\section{Upper zone cystic lung disease in HIV related Pneumocystis carinii pneumonia}

\author{
N C Cowan, J Moxham
}

\begin{abstract}
Although unusual, focal upper zone air space opacification and cyst formation in Pneumocystis carinii pneumonia and HIV infection can occur and may mimic "classical" tuberculosis.
\end{abstract}

(Thorax 1993;48:869-870)

Pneumocystis carinii pneumonia (PCP) is the most common HIV related pulmonary infection in both Great Britain and the USA. We report a patient with upper zone opacification and cavitatory disease caused by PCP in HIV disease mimicking "classical" tuberculosis. This is clinically important as tuberculosis is a major infectious pulmonary complication of HIV infection. Because of large overlap of the clinical and radiological signs in HIV related conditions, it is important to reach a definitive diagnosis in HIV infection.

\section{Case report}

A 37 year old man presented with a four month history of a non-productive cough, progressive exertional dyspnoea, and night sweats. Recent travel abroad had included a four month visit to Florida, USA. The patient looked well. Small lymph nodes were palpable in the left axilla. The presentation chest radiograph showed bilateral diffuse upper zone airspace opacification with widespread cavitation. Both hila were elevated but there was no hilar lymphadenopathy or pleural effusion (fig 1). A preliminary diagnosis of tuberculosis was made and antituberculosis chemotherapy was started. Results of further investigations at presentation were: $\mathrm{Hb} 10.9 \mathrm{~g} / \mathrm{dl}$, WBC $6.3 \times 10^{9} / 1$, granulocytes $4.7 \times 10^{9} / 1, \quad$ lymphocytes $1.3 \times 10^{9} / 1$, platelets $347 \times 10^{9} / 1$. Urea and electrolyte levels and liver function test results were normal. The CD4 count was $123 / \mathrm{mm}^{3}$ $\left(410-1540 / \mathrm{mm}^{3}\right)$, and the CD8 count $728 / \mathrm{mm}^{3}\left(230-1100 / \mathrm{mm}^{3}\right)$. The patient was HIV antibody positive. A bronchoscopy was performed but no acid fast bacilli, $P$ carinii or other organisms were identified in bronchial washings.

Despite antituberculosis chemotherapy the condition of the patient deteriorated rapidly over the following five days. He developed a high swinging pyrexia, tachycardia and

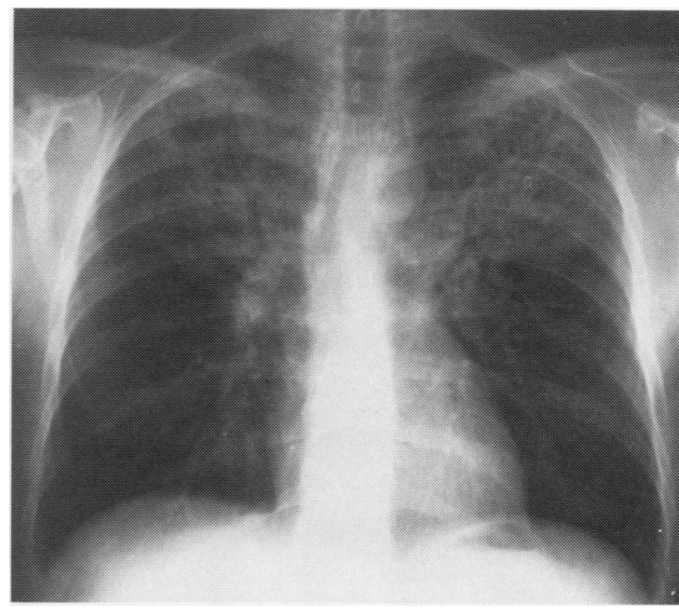

Figure 1 Presentation posteroanterior chest radiograph showing bilateral diffuse upper zone airspace opacification combined with subtle cystic change.

tachypnoea, with marked central cyanosis and hypoxia $\left(\mathrm{SaO}_{2}\right.$ on air $\left.66 \%\right)$. The chest radiograph now showed an increase in the density and extent of the airspace opacification involving the upper and mid zones of both lungs. A further bronchoscopy with bronchoalveolar lavage was performed. The patient was considered too ill for transbronchial biopsy. The endobronchial mucosa appeared normal and no organisms were identified in the bronchial washings. An open lung biopsy was therefore performed and histological examination showed an interstitial pneumonitis in which alveolar septa were extensively infiltrated by mononuclear and plasma cells. In the smaller airways $P$ carinii organisms were seen with Grocott staining. No fungi or acid fast bacilli were identified. Intravenous co-trimoxazole and hydrocortisone were commenced and the antituberculosis chemotherapy stopped. The patient made a gradual recovery over a period of three weeks and was discharged from hospital feeling well.

High resolution computed tomographic examination of the thorax performed after treatment showed the size and distribution of the lung cysts (fig 2).

\section{Discussion}

PCP is the most common HIV related pulmonary infection in Great Britain and the USA, occurring in $60-80 \%$ of patients with AIDS. ${ }^{1}$ Typically it presents as bilateral perihilar and/or basal reticular, reticulonodular or ground glass opacities which progress in 2-7 days to diffuse airspace opacification, initially sparing the apices but finally progressing to complete consolidation of the entire lung. ${ }^{2}$ Atypical patterns are reported in less than $5 \%$ of patients in most series and include unilateral focal consolidation, cystic disease, predominant upper lobe involvement, localised nodular densities, solitary pulmonary nodules, hilar and mediastinal lymphadenopathy, and pleural effusions. These atypical findings should initially suggest an alternative diagnosis. For example, hilar and mediastinal 


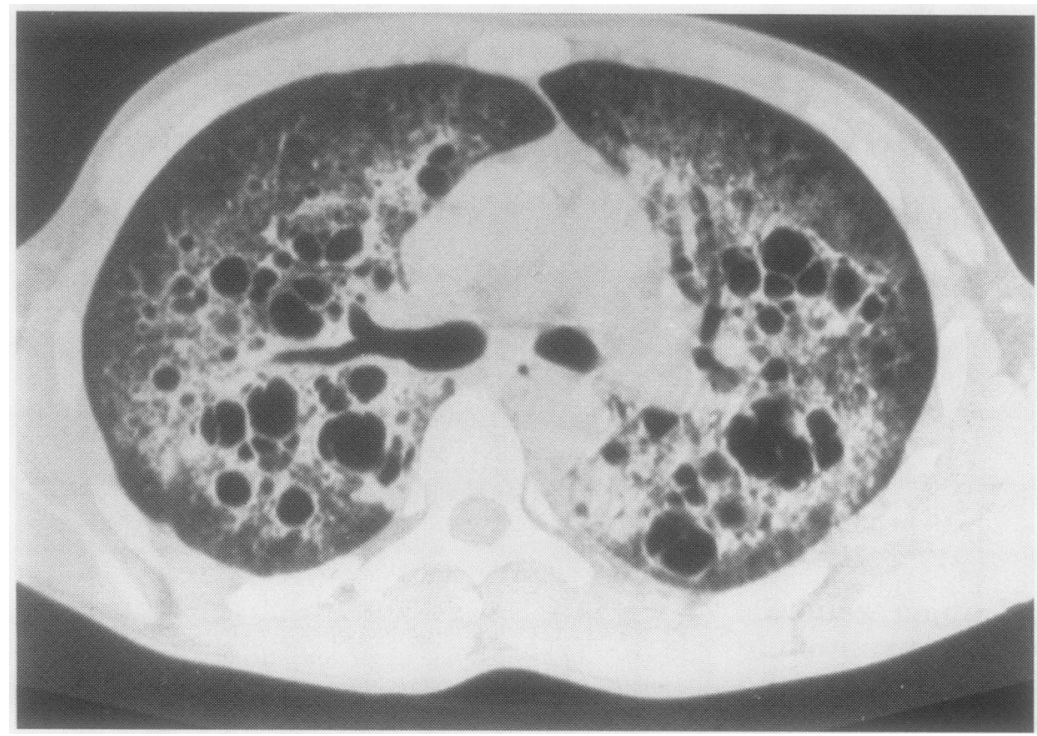

Figure 2 High resolution computed tomographic section of the chest showing multiple lung cysts with surrounding airspace opacification in the upper lobes and apical segments of the lower lobes. sufficient concentration to prevent the development of upper lobe PCP.

Fibreoptic bronchoscopy is the procedure of choice for diagnosing pulmonary opportunistic infections in HIV seropositive patients. The relative sensitivity of bronchoalveolar lavage and transbronchial biopsy for detecting $P$ carinii varies between series'; however, bronchoalveolar lavage has been reported to be the more effective method $\overline{3}$ because at least $50 \mathrm{~cm}^{3}$ of lung is sampled $\bar{\partial}$ compared with less than $1 \mathrm{~cm}^{3}$ by transbronchial biopsy, ${ }^{9}$ and the organisms lie almost entirely within the alveolar space. Fibreoptic bronchoscopy and transbronchial biopsy have a higher complication rate in HIV seropositive patients than in the general population. ${ }^{10}$ Complications are most commonly associated with transbronchial biopsy and include haemorrhage and pneumothorax, particularly in patients whose lungs are infected with $P$ carinii. ${ }^{10}$ Because of the rapid $\infty$ deterioration of our patient, the relatively low yield of additional diagnoses produced by transbronchial biopsy, and the risk of complications, transbronchial biopsy was not performed before open lung biopsy. An increased sensitivity and specificity of the bronchoalveolar lavage fluid for $P$ carinii might have been achieved by the use of indirect fluorescent antibodies or DNA amplification with ethidium bromide staining, but at the time these techniques were not available. $^{10}$

1 Naidich DP, McGuinness G. Pulmonary manifestations of AIDS. CT and radiographic correlations. Radiol Clin North Am 1991;29:999-1017.

2 Naidich DP, Garay SM, Goodman PC, Mybak BJ, Kramer EL. Pulmonary manifestations of AIDS. In Federle M, Megibow A, Naidich DP, eds. Radiology of acquired immune deficiency syndrome. New York: Raven Press, 1988:47-53.

3 Cowan NC, Salisbury J, Moxham J. Intrathoracic lymphadenopathy and HIV infection. $\mathrm{Br} \mathcal{F}$ Radiol 1992; 65:940-2.

4 Sandhu SJ, Goodman PC. Pulmonary cysts associated with Pneumocystis carinii pneumonia in patients with AIDS. Radiology 1989;173:33-5.

5 Feuerstein IM, Archer A, Pluda JM, Francis PS, Falloon $\mathrm{J}$, Masur $\mathrm{H}$, et al. Thin-walled cavities, cysts and pneumothorax in pneumocystic pneumonia: further observations with histopathologic correlation. Radiology observations with histopathologic correlation. Radiology 1990;174:697-

6 Milligan SA, Stulbarg MS, Gamsu G, Golden JA. Pneumocystis carinii pneumonia radiographically simulat- $N$ ing tuberculosis. Am Rev Respir Dis 1985;132:1124-6.

7 Murray JF. The white plague: down and out, or up and $N$ coming? Am Rev Respir Dis 1989;140:1788-95.

8 Chaffey MH, Klein SJ, Gamsu G, Blane P, Golden JA. Radiographic distribution of PCP in patients with AIDS treated with prophylactic inhaled pentamidine. Radiology 1990;175:715-9.

9 Griffiths MH, Kocjan G, Miller RF, Godfrey-Faussett P. Diagnosis of pulmonary disease in human immunodeficiency virus infection: role of transbronchial biopsy and bronchoalveolar lavage. Thorax 1989;44:554-8.

10 Miller RF, Millar AB, Weller IVD, Semple SJG. Empirical treatment without bronchoscopy for Pneumocystis carinii pneumonia in the acquired immunodeficiency syndrome. Thorax 1989;44:559-64. possible explanation is that aerosolised
pentamidine fails to reach the upper lobes in 